\title{
COLLECTIONS OF SEQUENCES HAVING THE RAMSEY PROPERTY ONLY FOR FEW COLOURS
}

\author{
Bruce M. Landman and Beata Wysocka
}

A family $\mathcal{C}$ of sequences has the $r$-Ramsey property if for every positive integer $k$, there exists a least positive integer $g^{(r)}(k)$ such that for every $r$-colouring of $\left\{1,2, \ldots, g^{(r)}(k)\right\}$ there is a monochromatic $k$-term member of $\mathcal{C}$. For fixed integers $m>1$ and $0 \leqslant a<m$, define a $k$-term $a(\bmod m)$-sequence to be an increasing sequence of positive integers $\left\{x_{1}, \ldots, x_{k}\right\}$ such that $x_{i}-x_{i-1} \equiv a(\bmod m)$ for $i=2, \ldots, k$. Define an $m$-a.p. to be an arithmetic progression where the difference between successive terms is $m$. Let $\mathcal{C}_{a(m)}^{*}$ be the collection of sequences that are either $a(\bmod m)$-sequences or $m$-a.p.'s. Landman and Long showed that for all $m \geqslant 2$ and $1 \leqslant a<m, \mathcal{C}_{a(m)}^{*}$ has the 2-Ramsey property, and that the 2-Ramsey function $g_{a(m)}^{(2)}(k, n)$, corresponding to $k$-term $a(\bmod m)$-sequences or $n$-term $m$-a.p.'s, has order of magnitude $m k n$. We show that $\mathcal{C}_{a(m)}^{*}$ does not have the 4-Ramsey property and that, unless $m / a=2$, it does not have the 3-Ramsey property. In the case where $m / a=2$, we give an exact formula for $g_{a(m)}^{(3)}(k, n)$. We show that if $a \neq 0$, there exist 4-colourings or 6-colourings (depending on $m$ and $a$ ) of the positive integers which avoid 2-term monochromatic members of $\mathcal{C}_{a(m)}^{*}$, but that there never exist such 3-colourings. We also give an exact formula for $g_{0(m)}^{(r)}(k, n)$.

\section{INTRODUCTION}

Many results in Ramsey theory take on the following general form: there exists a positive integer $f(r)$ such that for every partition of $[1, f(r)]=\{1, \ldots, f(r)\}$ into $r$ classes, some class will contain a set with property $P$ (where $P$ is some specified property). This can also be described by saying that for every $r$-colouring of $[1, f(r)]$, there is a monochromatic set with property $P$. Two of the most famous theorems of this type are van der Waerden's theorem [9] and Schur's theorem [8]. Schur's theorem says that for every positive integer $r$, there exists a positive integer $f(r)$ such that whenever $[1, f(r)]$ is $r$-coloured, there is a monochromatic set $\{x, y, z\}$ such that $x+y=$ $z$. Van der Waerden's theorem states that for all positive integers $k$ and $r$, there exists a positive integer $w(k, r)$ such that whenever $[1, w(k, r)]$ is $r$-coloured, there is a monochromatic $k$-term arithmetic progression. Estimation of the van der Waerden

Received 31st January, 1996

Copyright Clearance Centre, Inc. Serial-fee code: 0004-9729/97 \$A2.00+0.00. 
numbers $w(k, r)$ remains one of the most intriguing (and presumably one of the most difficult) problems in Ramsey theory, even for $r=2$ (see [2] for an in depth discussion). In recent years, several problems similar to van der Waerden's theorem have been looked at, where the family of $k$-term arithmetic progressions is replaced by some other family of $k$-term sequences. Examples can be found in $[1,3,4,5,6,7]$.

Let us say that a family $\mathcal{C}$ of sequences has the $r$-Ramsey property if for every positive integer $k$ there exists a positive integer $g^{(r)}(k)$ such that whenever $\left[1, g^{(r)}(k)\right]$ is $r$-coloured there is a monochromatic $k$-term member of $\mathcal{C}$. Thus van der Waerden's theorem tells us that the family of arithmetic progressions has the $r$-Ramsey property for every positive integer $r$. Schur's theorem, although it does not involve the parameter $k$, also is a result which holds for any number of colours $r$. Likewise, most other wellknown results in Ramsey theory which say that in a large enough set there exists some specified monochromatic structure, are true regardless of the number of colours used. In this paper we examine the $r$-Ramsey property for certain collections of sequences that were shown to be 2-Ramsey in [7], and show that these collections do not have the 4-Ramsey property, and in most cases do not have the 3-Ramsey property. We find this particularly intriguing because the Ramsey function corresponding to $k$-term sequences of this type, where two colours are used, grows only like a quadratic in $k$. This is in contrast to the van der Waerden function for 2 colours, $w(k, 2)$, for which the best known upper bound is enormous [2]; yet the collection of arithmetic progressions does have the $r$-Ramsey property for all $r$.

Let $m$ and $a$ be fixed integers such that $m \geqslant 2$ and $0 \leqslant a<m$. Define a $k$ term $a(\bmod m)$-sequence to be an increasing sequence of positive integers $\left\{x_{1}, \ldots, x_{k}\right\}$ such that $x_{i}-x_{i-1} \equiv a(\bmod m)$ for $2 \leqslant i \leqslant k$. Let $\mathcal{C}_{a(m)}$ denote the family of all $a(\bmod m)$-sequences. Define an $m$-a.p. to be an arithmetic progression such that the difference between consecutive terms is $m$. Denote by $\mathcal{C}_{a(m)}^{*}$ the family of all sequences that are either $a(\bmod m)$-sequences or $m$-a.p.'s. In [7] the authors showed that for all $m$ and all $a \neq 0$, the family $\mathcal{C}_{a(m)}$ does not have the 2-Ramsey property, but that $\mathcal{C}_{a(m)}^{*}$ does. If we let $g_{a(m)}(k)$ denote the least positive integer $N$ such that every 2-colouring of $[1, N]$ yields a monochromatic member of $\mathcal{C}_{a(m)}^{*}$, it was shown that $g_{a(m)}(k)=m k^{2}(1+o(1))$. In fact, the following more general result was found:

Theorem 1. (Landman and Long) Let $m, k, n \geqslant 2$ and $0 \leqslant a<m$. Let $g_{a(m)}(k, n)$ denote the least positive integer $N$ such that every 2-colouring of $[1, N]$ contains either a monochromatic $k$-term $a(\bmod m)$-sequence or a monochromatic $n$ term $m$-a.p. Then for all $1 \leqslant a<m, g_{a(m)}(k, n) \sim m k n$ (as $k \rightarrow \infty$ and $n \rightarrow \infty$ ). Also, $g_{0(m)}(k, n)=2 m(k-1)+1$.

Let us extend the notation of Theorem 1 so that $g_{a(m)}^{(r)}(k, n)$ denotes the least pos- 
itive integer $N$ (if it exists) such that every $r$-colouring of $[1, N]$ contains a monochromatic $k$-term $a(\bmod m)$-sequence or a monochromatic $n$-term $m$-a.p. If no such $N$ exists, we write $g_{a(m)}^{(r)}(k, n)=\infty$. In Section 2 we show that if $a>0$ and $m / a \neq 2$, then $g_{a(m)}^{(3)}(k, 2)=\infty$ for $k$ sufficiently large; thus, the family $\mathcal{C}_{a(m)}^{*}$ does not have the 3-Ramsey property. We also prove that if $m / a=2$, then this family does have the 3-Ramsey property, and we give an exact formula for $g_{a(m)}^{(3)}(k, n)$ (having order of magnitude $3 m n k$ ).

In Section 3 we consider $r>3$. We show that for all $1 \leqslant a<m$, the family $\mathcal{C}_{a(m)}^{*}$ does not have the $r$-Ramsey property if $r>3$. Further, we show that there exist 4-colourings or 6-colourings (depending on $m$ and $a$ ) of the positive integers that in fact avoid any 2-term monochromatic members of $\mathcal{C}_{a(m)}^{*}$, but that no such 3-colourings exist. In addition, the result of Theorem 1 which deals with the case of $a=0$ is easily extended to $r$ colours.

\section{Three Colors}

The following lemma will be useful in obtaining results throughout this paper.

Lemma 1. Let $c$ be a positive integer, $r \geqslant 2, m \geqslant 2$ and $0 \leqslant a<m$. Then

$$
g_{\mathrm{ca}(\mathrm{cm})}^{(r)}(k, n)=c\left(g_{a(m)}^{(r)}(k, n)-1\right)+1
$$

Proof: Let $N=g_{a(m)}^{(r)}(k, n)$. Now if $y$ is a positive integer, then $\left\{x_{i}: 1 \leqslant i \leqslant k\right\}$ is an $a(\bmod m)$-sequence if and only if $\left\{c x_{i}+y: 1 \leqslant i \leqslant k\right\}$ is a $c a(\bmod c m)$-sequence; and $\left\{x_{i}: 1 \leqslant i \leqslant k\right\}$ is an $m$-a.p. if and only if $\left\{c x_{i}+y: 1 \leqslant i \leqslant k\right\}$ is a $c m$-a.p. Hence, by the definition of $N$, any $r$ colouring of $\{1, c+1,2 c+1, \ldots,(N-1) c+1\}$ must contain a $k$-term monochromatic $c a(\bmod c m)$-sequence or an $n$-term monochromatic ca-a.p. Hence $g_{c a(c m)}^{(r)}(k, n) \leqslant c(N-1)+1$.

On the other hand, we know there is an $r$-colouring $\chi$ of $[1, N-1]$ that contains no monochromatic $k$-term $a(\bmod m)$-sequence and no monochromatic $n$-term $m$-a.p. Define $\chi^{\prime}$ on $[1, c(N-1)]$ by

$$
\chi^{\prime}([c(x-1)+1, c x])=\chi(x) \text { for } x=1, \ldots, N-1 .
$$

To complete the proof we shall show that $\chi^{\prime}$ avoids monochromatic $k$-term $c a(\bmod c m)$ sequences and monochromatic $n$-term $\mathrm{cm}$-a.p.'s. Assume, by way of contradiction, that $\left\{s_{i}\right\}$ is a monochromatic (with respect to $\chi^{\prime}$ ) sequence of one of these types. Let $t_{i}=\left\{s_{i} / c\right\rceil$ for each $i$. Then, by the reasoning of the previous paragraph, $\left\{t_{i}\right\}$ is either a $k$-term $a(\bmod m)$-sequence or an $n$-term $m$-a.p. that is monochromatic with respect to $\chi$, a contradiction. 
We now show that for most choices of $m$ and $a, \mathcal{C}_{a(m)}^{*}$ does not have the 3-Ramsey property (hence it has the $r$-Ramsey property only for $r=2$ ).

Theorem 2. Let $1 \leqslant a<m$ where $m / a \neq 2$ and let $s=\lceil(2 m / 3)\rceil$. Then $g_{a(m)}^{(3)}(s+1,2)=\infty$, so that $\mathcal{C}_{a(m)}^{*}$ does not have the 3-Ramsey property.

Proof: Let $d=\operatorname{gcd}(m, a)$. By Lemma 1 we know that $g_{a(m)}^{(3)}(k, n)$ $=d\left(g_{(a / d)(m / d)}^{(3)}(k, n)-1\right)+1$. Hence we may assume that $d=1$. To prove the theorem we provide a 3-colouring of the positive integers that contains no monochromatic 2 -term $m$-a.p. and no monochromatic $(s+1)$-term $a(\bmod m)$-sequence.

For each positive integer $x$, define $\bar{x}$ to be the element of $[1,2 m]$ such that $x \equiv$ $\bar{x}(\bmod 2 m)$. Let $t=\lceil(4 m / 3)\rceil$. Define $\chi$ to be the $2 m$-periodic colouring of the positive integers with $\chi(x)=\chi(\bar{x})$ for all $x$, where $\chi([1, s])=1, \chi([s+1, t])=2$, and $\chi([t+1,2 m])=3$. In other words, $\chi=I J K I J K I J K \ldots$, where $I=11 \ldots 1$ has length $s, J=22 \ldots 2$ has length $t-s$, and $K=33 \ldots 3$ has length $2 m-t$.

To see that there is no monochromatic 2-term $m$-a.p., note that since $m \geqslant 3$, we have $s<m<t$. Hence if $\bar{x} \in[1, s]$, then $s+1 \leqslant \overline{x+m} \leqslant 2 m$, so that $\chi(x+m) \neq$ $\chi(x)$. Likewise if $\bar{x} \in[s+1, t]$, then $\overline{x+m} \notin[s+1, t]$, and if $\bar{x} \in[t+1,2 m]$, then $\overline{x+m} \notin[t+1,2 m]$. Thus in all cases $\chi(x+m) \neq \chi(x)$, so that there is no monochromatic 2-term $m$-a.p.

To complete the proof, assume $X$ is an $(s+1)$-term $a(\bmod m)$-sequence. Then $X$ is of the form $\left\{x, x+a+j_{1} m, x+2 a+j_{2} m, \ldots, x+s a+j_{s} m\right\}$ where $j_{1} \leqslant \cdots \leqslant j_{s}$ are nonnegative integers. Since $d=1$ and $s<m$, from elementary group theory we know that the set

$$
\bar{X}=\left\{\bar{x}, \overline{x+a+j_{1} m}, \overline{x+2 a+j_{2} m}, \ldots, \overline{x+s a+j_{s} m}\right\}
$$

consists of $s+1$ distinct elements modulo $m$. Then these $s+1$ elements are also distinct modulo $2 m$ and, since each of the sets $[1, s],[s+1, t]$, and $[t+1,2 m]$ contains no more than $s$ elements, $X$ cannot be monochromatic under $\chi$. Hence there is no monochromatic $(s+1)$-term $a(\bmod m)$-sequence.

When $1 \leqslant a<m$, then the only circumstance under which $\mathcal{C}_{a(m)}^{*}$ has the 3Ramsey property is when $a=m / 2$. In the next theorem we establish this fact by giving a precise formula for the associated Ramsey function $g_{(m / 2)(m)}^{(3)}(k, n)$.

ThEOREM 3. Let $m>1$ be even, and let $k, n \geqslant 2$. Then

$$
g_{\frac{m}{2}(m)}^{(3)}(k, n)=\frac{m}{2}(3 k-5)(2 n-1)+1
$$

PROOF: We first prove the theorem for $m=2$. To show that (when $m=2$ ) the right-hand side of $(1)$ is a lower bound for $g_{1(2)}^{(3)}(k, n)$, we shall give a 3 -colouring of 
$[1,(3 k-5)(2 n-1)]$ that contains no monochromatic $k$-term $1(\bmod 2)$-sequence and no monochromatic $n$-term 2-a.p.

For $i=0, \ldots, 3 k-6$, define $I_{i}=[i(2 n-1)+1,(i+1)(2 n-1)]$. Hence

$$
\bigcup_{i=0}^{3 k-6} I_{i}=[1,(3 k-5)(2 n-1)] \text {. }
$$

If $k=2$, colour $I_{0}=[1,2 n-1]$ with the colouring $1212 \ldots 123$ (each of the colours 1 and 2 occurs $n-1$ times). This colouring clearly contains no 2 -term monochromatic $1(\bmod 2)$-sequence and no $n$-term monochromatic 2-a.p.

If $k \geqslant 3$, colour the $I_{i}$ as follows:

$$
\chi\left(I_{i}\right)= \begin{cases}\underbrace{1212 \ldots 12}_{2 n-2} 3 & \text { if } i \equiv 0(\bmod 3) \\ \underbrace{3131 \ldots 31}_{2 n-2} 2 & \text { if } i \equiv 1(\bmod 3) \\ \underbrace{2323 \ldots 23}_{2 n-2} 1 & \text { if } i \equiv 2(\bmod 3)\end{cases}
$$

Since $\left|I_{i}\right|=2 n-1$, we see that there is no monochromatic n-term 2-a.p.

Note that for each $j=0, \ldots, k-3$, the interval $I_{3 j} \cup I_{3 j+1} \cup I_{3 j+2}$ contains no 2 -term $1(\bmod 2)$-sequence having colour 1 . Hence any $1(\bmod 2)$-sequence with colour 1 that is contained in $\bigcup_{i=0}^{3 k-7} I_{i}$ has length at most $k-2$. Thus any $1(\bmod 2)$-sequence with colour 1 that is contained in $\bigcup_{i=0}^{3 k-6} I_{i}$ has length at most $k-1$.

Note also that if $k \geqslant 4$, then for each $j=0, \ldots, k-4$, the interval $I_{3 j+2} \cup I_{3 j+3} \cup$ $I_{3 j+4}$ contains no 2 -term $1(\bmod 2)$-sequence having colour 2 . Hence any $1(\bmod 2)$ sequence with colour 2 that is contained in $\bigcup_{i=2}^{3 k-8} I_{i}$ has length at most $k-3$. Since $I_{0} \cup I_{1}$ and (for $\left.k \geqslant 3\right) I_{3 k-7} \cup I_{3 k-6}$ can each contain at most one term of a 1 (mod $2)$-sequence having colour 2 , we see that any $1(\bmod 2)$-sequence having colour 2 that is contained in $\bigcup_{i=0}^{3 k-6} I_{i}$ has length at most $k-1$.

Finally, we see that for each $j=0, \ldots, k-3$, the interval $I_{3 j+1} \cup I_{3 j+2} \cup I_{3 j+3}$ contains no 2 -term $1(\bmod 2)$-sequence having colour 3 . Therefore, any $1(\bmod 2)$ sequence with colour 3 that is contained in $\bigcup_{i=1}^{3 k-6} I_{i}$ has length at most $k-2$. Thus any $1(\bmod 2)$-sequence having colour 3 that is contained in $\bigcup_{i=0}^{3 k-6} I_{i}$ has length at most
$k-1$.

We have shown that $\chi$ avoids both monochromatic $k$-term $1(\bmod 2)$-sequences and monochromatic $n$-term 2-a.p.'s, and therefore $g_{1(2)}^{(3)}(k, n) \geqslant(3 k-5)(2 n-1)+1$. 
Next we prove that

$$
g_{1(2)}^{(3)}(k, n) \leqslant(3 k-5)(2 n-1)+1 .
$$

Let $I(k, n)=[1,(3 k-5)(2 n-1)+1]$. We need to show that for every 3-colouring of $I(k, n)$, there is either a monochromatic $k$-term $1(\bmod 2)$-sequence or a monochromatic n-term 2-a.p.

Given a 3-colouring $\phi$ of $I(k, n)$, let $a_{i}(\phi)$ denote the length of the longest monochromatic $1(\bmod 2)$-sequence that has colour $i(i=1,2,3)$. Let $S(k, n, \phi)=$ $a_{1}(\phi)+a_{2}(\phi)+a_{3}(\phi)$. To prove (2) our strategy is to establish the following fact:

FACT 1. If $\phi$ is a 3-colouring of $I(k, n)$ for which there is no monochromatic $n$-term 2-a.p., then $S(k, n, \phi) \geqslant 3(k-1)+1$.

Then (2) will follow easily from Fact 1 by the pigeon-hole principle, since there is either a monochromatic $n$-term 2 -a.p. or a monochromatic $k$-term $1(\bmod 2)$-sequence.

We prove Fact 1 by induction on $k$. Let $k=2$ and let $\phi$ be any 3-colouring of $[1,2 n]$ with no monochromatic $n$-term 2-a.p. So there are odd numbers $o_{1}, o_{2}$ and even numbers $e_{1}, e_{2}$ in $[1,2 n]$ such that $\phi\left(o_{1}\right) \neq \phi\left(o_{2}\right)$ and $\phi\left(e_{1}\right) \neq \phi\left(e_{2}\right)$. Since there are only three colours, $\phi\left(e_{i}\right)=\phi\left(o_{j}\right)$ for some $i$ and $j, 1 \leqslant i, j \leqslant 2$. Therefore $\left\{e_{i}, o_{j}\right\}$ is a monochromatic 2 -term $1(\bmod 2)$-sequence, say of colour 1 . We also see (since there is no monochromatic $n$-term 2-a.p.) that there is some even number $e$ and some odd number $o$ such that $\phi(e) \neq 1$ and $\phi(o) \neq 1$. Hence either $\phi(o)=\phi(e)$ and we have another monochromatic 2 -term $1(\bmod 2)$-sequence, or else $\{e\}$ and $\{o\}$ are each 1 -term $1(\bmod 2)$-sequences, not of the same colour, and neither having colour 1 . In either case, $S(2, n, \phi) \geqslant 4$.

Now assume $k \geqslant 2$ and that Fact 1 holds for $k$. Let $\phi$ be any 3 -colouring of $I(k+1, n)=[1,(3 k-2)(2 n-1)+1]$ such that there is no monochromatic $n$-term 2-a.p. To complete the proof we show that $S(k+1, n, \phi) \geqslant 3 k+1$.

Let $A_{1}=[(3 k-5)(2 n-1)+1,(3 k-4)(2 n-1)+1], A_{2}=[(3 k-4)(2 n-1)+$ $1,(3 k-3)(2 n-1)+1]$, and $A_{3}=[(3 k-3)(2 n-1)+1,(3 k-2)(2 n-1)+1]$. Thus, $I(k+1, n)=I(k, n) \cup A_{1} \cup A_{2} \cup A_{3}$. For each $j(j=1,2,3)$, since $A_{j}$ has length $2 n$, by the same argument used for $[1,2 n]$ in the case of $k=2, A_{j}$ contains a monochromatic sequence $Y_{j}=\left\{e_{j}, o_{j}\right\}$, where $e_{j}$ is even and $o_{j}$ is odd. Let $\bar{\phi}$ represent the colouring $\phi$ restricted to the interval $I(k, n)$. Then, by using the contributions of the $Y_{j}$, we have that $S(k+1, n, \phi) \geqslant S(k, n, \bar{\phi})+3$. Therefore, by the inductive hypothesis, $S(k+1, n, \phi) \geqslant 3 k+1$.

We have established the theorem for $m=2$. Now let $m$ be any positive even integer. Then by this result for $m=2$, and Lemma 1 , we have

$$
g_{\frac{m}{2}(m)}^{(3)}(k, n)=\frac{m}{2}\left(g_{1(2)}^{(3)}(k, n)-1\right)+1=\frac{m}{2}(3 k-5)(2 n-1)+1,
$$


and the proof is complete.

\section{MORE THAN THREE COLOURS}

From the results of Section 2, we know that if $1 \leqslant a<m$ and $m / a \neq 2$, then $\mathcal{C}_{a(m)}^{*}$ does not have the $r$-Ramsey property whenever $r \geqslant 3$. The remaining cases are those where either (i) $a=0$; or (ii) $r \geqslant 4$ and $m / a=2$.

The case of $a=0$ is a unique case, and is also easier (one explanation for this is that when $a>0$, an $a(\bmod m)$-sequence can be thought of as an arithmetic progression modulo $m$, while a $0(\bmod m)$-sequence cannot be considered as part of the collection of arithmetic progression's modulo $m$ ). Note by the following theorem that the value of $g_{0(m)}^{(r)}(k, n)$ is independent of $n$.

Theorem 4. For all $r, m, k, n \geqslant 2, g_{0(m)}^{(r)}(k, n)=r m(k-1)+1$.

PROOF: In $[1, r m(k-1)+1]$ there are $r(k-1)+1$ integers that are congruent to $1(\bmod m)$. Under any $r$-colouring of $[1, r m(k-1)+1]$ at least $k$ of these integers must be monochromatic, giving a $k$-term monochromatic $0(\bmod m)$-sequence. Hence $g_{0(m)}^{(r)}(k, n) \leqslant r m(k-1)+1$.

To show the reverse inequality, let $\chi$ be the following $r$-colouring of $[1, r m(k-1)]$ :

$$
\underbrace{11 \ldots 1}_{m} \underbrace{22 \ldots 2}_{m} \cdots \underbrace{r r \ldots r}_{m} \underbrace{11 \ldots 1}_{m} \underbrace{22 \ldots 2}_{m} \cdots \underbrace{r r \ldots r}_{m} \cdots \cdots \cdots \underbrace{11 \ldots 1}_{m} \underbrace{22 \ldots 2}_{m} \cdots \underbrace{r r \ldots r}_{m}
$$

where there are $r(k-1)$ blocks of size $m$. Then $\chi$ avoids monochromatic $k$-term $0(\bmod m)$-sequences. Also, since $n \geqslant 2, \chi$ avoids monochromatic $n$-term $m$-a.p.'s, and the proof is complete.

For the only remaining case, $m / a=2$ and $r \geqslant 4$, it turns out that $\mathcal{C}_{a(m)}^{*}$ does not have the $r$-Ramsey property. In fact, the next theorem gives the stronger result that, whenever $m /(\operatorname{gcd}(a, m))$ is even, it is possible to 4 -colour the positive integers so as to avoid monochromatic 2 -term members of $\mathcal{C}_{a(m)}^{*}$.

THEOREM 5. Let $1 \leqslant a<m$ and let $m / d$ be even where $d=\operatorname{gcd}(m, a)$. Then $g_{a(m)}^{(4)}(2,2)=\infty$.

Proof: By Lemma 1 it is sufficient to prove the result when $d=1$. Hence we assume that $m$ is even and $a$ is odd. We give a colouring of the positive integers that contains no monochromatic 2 -term $a(\bmod m)$-sequences and no monochromatic 2 -term m-a.p.'s.

Let $Q$ be the string $1212 \ldots 12$ of length $m$, and let $R$ be the string $3434 \ldots 34$ of length $m$. Colour the positive integers with the colouring $\chi=Q R Q R Q R \ldots$. Clearly $\chi$ contains no monochromatic 2 -term $m$-a.p. Also, since $m$ is even, there is no pair of 
positive integers $x$ and $y$ such that $y-x$ is odd and $\chi(x)=\chi(y)$. Thus there is no monochromatic 2-term $a(\bmod m)$-sequence.

By Theorem 3 we know that we cannot replace the value of $r=4$ with $r=3$ in Theorem 5 and still have a true statement. Looking at Theorem 2 one might wonder whether there are any values of $m$ and $a$ for for which $g_{a(m)}^{(3)}(2,2)=\infty$. The next theorem shows that this is not the case.

TheOREM 6. Let $1 \leqslant a<m$. Then $g_{a(m)}^{(3)}(2,2) \leqslant 3 m$.

ProOF: Assume the statement is false. Hence for some $a$ and $m$ there exists a 3colouring $\chi:[1,3 m] \rightarrow\{1,2,3\}$ having no monochromatic 2-term $a(\bmod m)$-sequence and no monochromatic 2-term $m$-a.p. Without loss of generality, let $\chi(m+1)=1$ and $\chi(2 m+1)=2$.

By assumption, $\chi(1) \neq \chi(m+a+1), \chi(m+1) \neq \chi(m+a+1)$, and $\chi(1) \neq$ $\chi(m+1)$. Hence we have two cases: (a) $\chi(1)=2$ and $\chi(m+a+1)=3$, and (b) $\chi(1)=3$ and $\chi(m+a+1)=2$. We prove only case (a), as the proof of case (b) is essentially the same. Now if $\chi(1)=2$ and $\chi(m+a+1)=3$, then $\chi(2 m+a+1)=1$, but then $\{m+1,2 m+a+1\}$ is a monochromatic $a(\bmod m)$-sequence, a contradiction.

Considering Theorems 2,3 , and 5, one would suspect that, for $a>0$ and $m / d$ odd, $g_{a(m)}^{(4)}(2,2)=\infty$. However, computer output suggests that, for $m / d$ odd, the least $r$ for which $g_{a(m)}^{(r)}(2,2)=\infty$, is always either 5 or 6 . We are able to show that for all $m \geqslant 2$ and $a \neq 0$ it is possible to 6 -colour the positive integers so as to avoid monochromatic 2 -term $a(\bmod m)$-sequences and monochromatic 2 -term $m$-a.p.'s.

THEOREM 7. Let $1 \leqslant a<m$ and let $m / d$ be odd where $d=\operatorname{gcd}(m, a)$. Then $g_{a(m)}^{(6)}(2,2)=\infty$.

Proof: By Lemma 1, we assume $d=1$. We provide a 6 -colouring of the positive integers that avoids monochromatic 2-term members of $\mathcal{C}_{a(m)}^{*}$. The proof splits naturally into two cases.

CASE 1. $a<m / 2$. Let $m=q a+t$, where $q$ is an integer and $0 \leqslant t<a$. We have two subcases.

(i) $q$ is even. Colour $[1,2 m]$ with the colouring

$$
A B A B \ldots A B \quad E^{\prime} \quad C D C D \ldots C D \quad F^{\prime \prime},
$$

where $A=11 \ldots 1, B=22 \ldots 2, C=33 \ldots 3$, and $D=44 \ldots 4$ each have length $a$, where $E^{\prime}=55 \ldots 5$ and $F^{\prime}=66 \ldots 6$ each have length $t$, and where each of $A, B, C, D$ occurs $q / 2$ times. Now extend this to a colouring of the positive integers by repeating it (that is, we now have a $2 m$-periodic colouring of the positive integers). Call this colouring of the positive integers $\chi$. 
There is no monochromatic 2-term $m$-a.p. with respect to $\chi$, for: if $i \in A$, then $i+m \in C$; if $i \in B$, then $i+m \in D$; if $i \in C$, then $i+m \in A$; et cetera.

Now let $\{x, y\}$ be an $a(\bmod m)$-sequence. Let $x=c m+j$, where $1 \leqslant j \leqslant m$. If $1 \leqslant j \leqslant m-a$, then we see that $\chi(x) \neq \chi(x+a)$ and $\chi(x) \neq \chi(x+m+a)$. Therefore $\chi(x) \neq \chi(y)$. If, instead, $m-a<j \leqslant m$, then $x$ belongs to some copy of $B, E^{\prime}, D$, or $F^{\prime}$, while $y$ belongs to some copy of $A$ or $C$. Thus $\{x, y\}$ is not monochromatic.

(ii) $q$ is odd. In this case colour $[1,2 m]$ with the colouring

$$
A B A B \ldots A B \quad E B^{\prime} \quad C D C D \ldots C D \quad F D^{\prime}
$$

where $A, B, C$, and $D$ are defined as in subcase (i) and each occurs $(q-1) / 2$ times, where $E=55 \ldots 5$ and $F=66 \ldots 6$ each have length $a$, and where $B^{\prime}=22 \ldots 2$ and $D^{\prime}=44 \ldots 4$ each have length $t$. As in subcase (i) extend this to a $2 m$-periodic colouring of the positive integers. Since $A B A B \ldots A B E B^{\prime}$ has length $m$, it is clear that there is no monochomatic 2-term $m$-a.p.

Let $\{x, y\}$ be an $a(\bmod m)$-sequence. If $x=c m+j$ where $1 \leqslant j \leqslant m-a$, then $\{x, y\}$ cannot be monochromatic for the same reason given in subcase (i). If $m-a<j \leqslant m$, then $x$ belongs to some copy of $E, B^{\prime}, F$, or $D^{\prime}$, while $y$ belongs to some copy of $A$ or $C$. This proves the theorem for the case in which $a<m / 2$.

CASE 2. $a>m / 2$. (Equality is impossible since $m / d$ is odd.) Let $b=m-a$. Let $m=q^{\prime} b+t^{\prime}$, where $q^{\prime}$ is an integer and $0 \leqslant t<b$. Again we have two subcases, depending on whether $q^{\prime}$ is even or odd. For convenience, we do the subcase in which $q^{\prime}$ is even, as the other is done in the same way. We use the same colouring that was used in Case 1, subcase (i), except replace $a$ by $b, q$ by $q^{\prime}$ and $t$ by $t^{\prime}$. Denote this new colouring by $\chi^{\prime}$. Then we know that $\chi^{\prime}$ will avoid monochromatic 2-term $m$-a.p.'s and monochromatic 2 -term $b(\bmod m)$-sequences. The proof will be completed by showing that there are also no monochromatic 2-term $a(\bmod m)$-sequences. Assume, by way of contradiction, that $\{x, y\}$ is a monochromatic $a(\bmod m)$-sequence under $\chi^{\prime}$. Let $h$ be even such that $x+h m>y$. Then $\{y, x+h m\}$ is a $b(\bmod m)$-sequence and, since $\chi^{\prime}$ is $2 m$-periodic, $\chi^{\prime}(x)=\chi^{\prime}(x+h m)$, so that $\{y, x+h m\}$ is a monochromatic $b(\bmod m)$-sequence, a contradiction.

We summarise the results on the Ramsey properties of $\mathcal{C}_{a(m)}^{*}$ in the following table. For the different choices of $m, a$, and $r$, we give the asymptotic value, as $k \rightarrow \infty$, of the associated Ramsey function $g_{a(m)}^{(r)}(k, k)$. 


\begin{tabular}{|c|c|c|c|}
\hline values of $a, m$ & $r=2$ & $r=3$ & $r \geqslant 4$ \\
\hline$m / a=2$ & $m k^{2}$ & $3 m k^{2}$ & $\infty$ \\
$m / a \neq 2, a \neq 0$ & $m k^{2}$ & $\infty$ & $\infty$ \\
$a=0$ & $2 m k$ & $3 m k$ & $r m k$ \\
\hline
\end{tabular}

Table 1. Asymptotic value of $g_{a(m)}^{(r)}(k, k)$

\section{REFERENCES}

[1] T.C. Brown, P. Erdös and A.R. Freedman, 'Quasi-progressions and descending waves', J. Combin. Theory Ser. A 53 (1990), 81-95.

[2] R.L. Graham, B.L. Rothschild, and J.H. Spencer, Ramsey theory, (2nd edition) (John Wiley and Sons, NY, 1990).

[3] R.N. Greenwell and B.M. Landman, 'On the existence of a reasonable upper bound for the van der Waerden numbers', J. Combin. Theory Ser. A 50 (1989), 82-86.

[4] B.M. Landman, 'An upper bound for van der Waerden-like numbers using $k$ colors,', Graphs Combin. 9 (1993), 177-184.

[5] B.M. Landman, 'Ramsey functions associated with second order recurrences', J. Combin. Math. Combin. Comput. 15 (1994), 119-128.

[6] B.M. Landman, 'Ramsey functions for quasi-progressions', Graphs Combin. (to appear).

[7] B.M. Landman and A.F. Long, 'Ramsey functions for sequences with adjacent differences in a specified congruence class', Congr. Numer. 103 (1994), 3-20.

[8] I. Schur, 'Uber die kongruenz $x^{n}+y^{n} \equiv z^{n}(\bmod p)$ ', J. ber. Deutsch. Math. Verein. 25 (1916), 114-116.

[9] B.L. van der Waerden, 'Beweis einer Baudetschen Vermutang', Nieuw Arch. Wisk. 15 (1927), 212-216.

Department of Mathematics Sciences

University of North Carolina at Greensboro

North Carolina 27412

United States of America 